\title{
Balkanologie
}

Balkanologie Revue d'études pluridisciplinaires

Vol. $16 n^{\circ} 1 \mid 2021$

Au-delà de la "route des Balkans ": mondes sociaux des circulations

\section{Reconstruction et résilience de la destination touristique bosnienne : le potentiel des nouvelles technologies}

Reconstruction and Resilience of the Bosnian Tourist Destination: The Potential

of New Technologies

\section{Cyril Martin-Colonna}

\section{OpenEdition}

Journals

Édition électronique

URL : https://journals.openedition.org/balkanologie/3203

DOI : 10.4000/balkanologie.3203

ISSN : 1965-0582

Éditeur

Association française d'études sur les Balkans (Afebalk)

Référence électronique

Cyril Martin-Colonna, « Reconstruction et résilience de la destination touristique bosnienne : le potentiel des nouvelles technologies », Balkanologie [En ligne], Vol. $16 n^{\circ} 1$ | 2021, mis en ligne le 01 juin 2021, consulté le 03 octobre 2021. URL : http://journals.openedition.org/balkanologie/3203 ; DOI : https://doi.org/10.4000/balkanologie.3203

Ce document a été généré automatiquement le 3 octobre 2021.

(c) Tous droits réservés 


\title{
Reconstruction et résilience de la destination touristique bosnienne : le potentiel des nouvelles technologies
}

\author{
Reconstruction and Resilience of the Bosnian Tourist Destination: The Potential \\ of New Technologies
}

Cyril Martin-Colonna

Le $\mathrm{xx}^{\mathrm{e}}$ siècle marque une apogée en termes de conflits et de destructions, notamment lors de la Seconde Guerre mondiale qui a vu des villes quasiment rasées, comme Coventry en Angleterre ou Dunkerque en France ${ }^{1}$. Durant cette période, la destruction des villes a connu une ampleur sans précédent ${ }^{2}$. Le $\mathrm{xxI}^{\mathrm{e}}$ siècle présente de nouveaux risques, au titre desquels la destruction des villes et des sociétés par les guerres et les menaces terroristes ${ }^{3}$. Pour tenter de faire face à ces nouveaux défis et risques, le concept de résilience, et plus particulièrement de résilience des villes, a été développé au début des années 2000 pour devenir un enjeu stratégique dans la préservation des modes de vie, dans la sauvegarde des populations, permettant d'éviter toute cassure dans la dynamique (économique, politique, sociale) pouvant mener à des crises majeures $^{4}$. En tourisme, le concept de résilience est également utilisé, particulièrement en ce qui concerne les efforts déployés par une destination pour se relancer après avoir subi des dommages importants à la suite de catastrophes naturelles, de crises sanitaires ou de risques sécuritaires ${ }^{5}$. Les différents acteurs publics et institutionnels élaborent des politiques réglementaires et mettent en œuvre les moyens financiers et humains nécessaires pour soutenir la reconstruction des sites touristiques impactés, afin de relancer la destination le plus rapidement possible ${ }^{6}$. Le tourisme suscite de nombreuses attentes, aussi bien de la part des gouvernements que des décideurs publics et privés, qui le considèrent comme « une source potentielle de croissance pour leur économie, d'activités aux dimensions fortement inclusives et d'amélioration du bien-être de leur population $»^{7}$. Malgré un modèle de tourisme de masse reposant sur les atouts naturels, 
culturels ou historiques, desservi selon des offres standardisées commercialisées par des tours opérateurs et des agences de voyages, les destinations touristiques transforment et varient leurs modèles pour faire face à la disparition progressive de la massification touristique au profit d'un tourisme intelligent priorisant la compétitivité de la destination par sa capacité à innover et à se renouveler ${ }^{8}$. Ces vingt dernières années, les technologies de l'information, la création de plateformes numériques de type peer-to-peer (Booking, Airbnb, Expedia) ou encore la réalité virtuelle au service des visites muséales et patrimoniales font exploser l'attractivité des destinations et les projets touristiques.

2 Faisant appel à des références stéréotypées afin d'attirer les visiteurs étrangers, la destination touristique bosnienne émerge dans les guides de voyage dès le $\mathrm{xIX}^{\mathrm{e}}$ siècle. En 1935, un ouvrage yougoslave suggère, par exemple, que les «cent mosquées » de Sarajevo et «les jardins du pittoresque Mostar forment un ensemble qui rappelle les contes des mille et une nuits $"$. Les Jeux olympiques de Sarajevo en 1984 marquent l'apogée de l'attractivité touristique du pays et de sa capitale, exploitant la symbolique de l'évènement sportif et de leur exposition médiatique, célébrant la richesse multiculturelle et le vivre ensemble. Depuis le début des années 1990, le secteur touristique bosnien a été affecté par différents facteurs endogènes et exogènes. Outre la guerre qui a mis à terre la destination Bosnie-Herzégovine, les années qui ont suivi ont été marquées par l'instabilité institutionnelle et ethnique découlant des accords de Dayton. La mise sous perfusion économique, à l'aide de multiples programmes d'aide internationaux et européens, a mis au second plan le processus de reconstruction de la destination touristique par les autorités fédérales. Malgré tout, le gouvernement bosnien, conscient du potentiel économique du tourisme et de son retard vis-à-vis des autres pays européens et de ses voisins proches tels que la Croatie ou la Serbie, a adopté un plan de développement stratégique du tourisme pour la période 2008-2018 comprenant cinq facteurs clés: environnement politique et sécuritaire stable, tendances économiques favorables, état satisfaisant de l'environnement, développement technologique approprié et situation sanitaire favorable ${ }^{10}$. Ce plan ne peut cependant être considéré que comme une base de travail. Les cinq piliers de développement touristiques mettent surtout en avant les problématiques et les fractures de l'organisation touristique et de facto institutionnelle de la gouvernance fédérale, plus qu'ils n'apportent de véritables objectifs chiffrés et des solutions concrètes. La reconstruction et la résilience de la destination touristique bosnienne par les nouvelles technologies font partie de cet ensemble de mesures visant à reconstruire un territoire touristique encore marqué par le conflit militaire. L'usage des technologies de l'information à des fins touristiques a favorisé le développement des destinations historiques mais aussi de destinations ayant connu des événements d'ordres humains, naturels ou économiques ${ }^{11}$, comme la Croatie ou la Slovénie. La gouvernance fédérale bosnienne, à la recherche de retombées économiques au détriment d'un réel plan de reconstruction de la destination, repose sur une collaboration multi-échelle entre les pouvoirs locaux, privés, transnationaux et associatifs pour attirer les fonds financiers nécessaires à l'élaboration de projets. En nous penchant sur différents projets technologiques et digitaux à visée touristique, nous verrons comment ces acteurs se sont emparés de cette initiative afin de la rendre concrète dans le temps et quels en sont les résultats sur le territoire. 
3 Nous nous intéresserons donc, dans ce texte, à l'émergence de projets touristiques basés sur le numérique et les nouvelles technologies en Bosnie-Herzégovine. Pour ce faire, nous analyserons deux projets prenant place à Sarajevo : le premier, qui s'intitule "The Sarajevo Survival Tools Virtual Environment ", met en avant à la fois le rôle de la réalité virtuelle comme promoteur culturel et historique dans la compréhension du siège de Sarajevo par les jeunes générations et les touristes ainsi que l'abandon des autorités dans la continuité du projet; le second, "Sarajevo Smart City Hackathon 2020 ", entend faire entrer la capitale dans l'ère de la ville intelligente, durable et inclusive pour les populations locales et touristiques par la collaboration des autorités locales, privées et onusienne.

\section{De la reconstruction à la résilience : un concept polysémique et un processus adaptatif}

4 Les conflits armés, comme celui qui a eu lieu en Bosnie-Herzégovine, obligent les décideurs locaux à repenser en profondeur le paysage touristique et, de facto, sa mise en tourisme $\mathrm{e}^{12}$ en lien avec les dégradations du patrimoine bâti et naturel, la perte de patrimoine immatériel unique ou encore la reconfiguration sociétale. Cette nouvelle mise en tourisme de la Bosnie-Herzégovine donne lieu à une reconstruction de l'offre touristique proposée avant le conflit (tourisme de nature, santé) axée sur les nouvelles technologies (réalité virtuelle, promotion et marketing touristique à l'attention des touristes internationaux, développement d'outils pour la ville intelligente) et au développement d'une nouvelle offre autour du patrimoine et de la mémoire du conflit ${ }^{13}$. Façonnée par les différents acteurs institutionnels et touristiques de la destination, la mise en tourisme devient porteuse de représentations diffusées et propagées par le tourisme, devenant un moyen d'améliorer les conditions de vie des habitants ${ }^{14}$. Que ce soit en villes, dans des métropoles ou des destinations touristiques, les risques d'être touchés par des évènements naturels ou anthropiques (aléas climatiques, risques naturels, évènements politiques ou sociaux violents, etc.) sont tout aussi importants, invitant les acteurs des destinations touristiques à réfléchir au concept de résilience touristique au sein de la résilience globale ${ }^{15}$. Si le succès du tourisme est notamment lié à la diversité et à la richesse des ressources dont disposent les destinations touristiques (ressources naturelles, climatiques, architecturales, culturelles, etc.) et à la qualité de leurs équipements et infrastructures, ceux-ci ne suffisent pas à pérenniser les destinations affectées par de tels risques ${ }^{16}$.

5 L'étude du cas de la Bosnie-Herzégovine se révèle un choix porteur pour analyser la résilience de la destination touristique face à une perturbation majeure dans la dynamique touristique. Suite au conflit, de nouvelles complexités se sont nouées en termes de représentation des lieux par les acteurs touristiques et urbains, qui sont ressenties par les visiteurs qui viennent découvrir ces lieux et les populations locales ${ }^{17}$. Le regard que leur portent les visiteurs est pluriel, complexe à appréhender ${ }^{18}$. Il entremêle quête d'originalité et d'altérité, prise de conscience humaniste et "résiliente", mais aussi processus d'identification ${ }^{19}$. Actuellement, l'industrie touristique bosnienne, en quête de retombées économiques, court le risque de n'être qu'un producteur d'imaginaires, fondés sur la partition identitaire et ethnique consécutive à la guerre ${ }^{20}$, reposant sur des fantasmes ainsi que sur des infrastructures vieillissantes et dépassées ne répondant pas aux normes touristiques actuelles. 
Cependant, de nouvelles initiatives, axées notamment sur les nouvelles technologies, se développent à différentes échelles (politiques, touristiques, publiques, privées, associatives, citoyennes...) à travers le pays.

6 Afin d'opérationnaliser le concept de résilience touristique, nous le définissons comme la capacité à s'adapter selon un processus intégrant des acteurs multiples "pour s'adapter aux menaces et atténuer ou éviter les dommages. La résilience peut être trouvée dans des bâtiments résistants aux aléas ou des systèmes sociaux adaptatifs ${ }^{21}$ ». Les différentes communautés ethniques du pays prennent acte de leur environnement naturel, physique ou social, et s'accommodent de son fonctionnement. De ce fait, la reconstruction du système touristique suite à une perturbation intègre la complexité même de la destination ${ }^{22}$. Dans le cas précis du tourisme et de la place du numérique et des nouvelles technologies, nous reprendrons la résilience selon le cycle d'adaptation panarchique de Holling, qui démontre que les systèmes sont fortement dynamiques et tout autant instables, pouvant subir des vagues de créativité évolutive, d'inertie et de dégringolade ${ }^{23}$. Dans le cycle adaptatif de Holling, consolidé par Harvey Lemmelin et al., quatre phases de développement constituent la résilience en tourisme: 1/ la phase de développement ; 2/ la phase de stabilité ; $3 /$ la phase de libération ou destruction créatrice; 4/ la phase de réorganisation. Ces quatre phases de développement permettent de mettre l'emphase sur la complexité adaptative de l'industrie touristique face aux évènements endogènes et exogènes. Outre la définition de Holling et Gunderson ${ }^{24}$, nous nous référons également à la définition de Cholat et al. ${ }^{25}$ qui voient en la résilience touristique,

l'ensemble des ajustements des pratiques et des activités développées par les différents acteurs concernés pour faire face à des pressions ou contraintes « externes» (changements environnementaux, nouveaux rythmes, insécurité, peurs, saturations...), «internes» (nouvelles attentes des consommateurs, modes, nouveaux besoins et comportements...), à des « aléas » (crise, accident...) ou pour exploiter les opportunités (nouvelles technologies, jeux, nouvelles formes d'hébergements ou de transports...) afin de rester attractif dans des conditions nouvelles.

Figure 1. Modèle de cycle adaptatif et panarchique des systèmes complexes

INCLUDEPICTURE $\quad$ "../../../../var/folders/9g/z6yww88s1nlbbyb0wf0w06vw0000gn/T/ com.microsoft.Word/WebArchiveCopyPasteTempFiles/img-1.jpg" $\backslash *$ MERGEFORMAT INCLUDEPICTURE " $/$ Users/../../../../var/folders/9g/ z6yww88s1nlbbyb0wf0w06vw0000gn/T/com.microsoft.Word/ WebArchiveCopyPasteTempFiles/img-1.jpg" ${ }^{*}$ MERGEFORMATINET INCLUDEPICTURE $" \backslash \backslash \backslash \backslash$ Users $\backslash \backslash . . \backslash . . \backslash \backslash . . \backslash . . \backslash \backslash$ var $\backslash \backslash$ folders $\backslash \backslash 9 g \backslash \backslash z 6 y w w 88 s 1$ nlbbyb0wf0w06vw0000gn $\backslash \backslash T \backslash$ \com.microsoft.Word $\backslash \backslash$ WebArchiveCopyPasteTempFiles \\img-1.jpg" MERGEFORMATINET INCLUDEPICTURE $\quad \backslash \backslash \backslash \backslash$ Users $\backslash \backslash . . \backslash$.. $\backslash \backslash . . \backslash . . \backslash \backslash$ var $\backslash \backslash$ folders $\backslash \backslash 9 \mathrm{~g} \backslash$

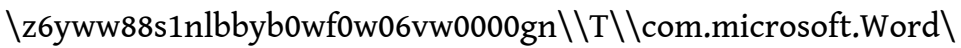




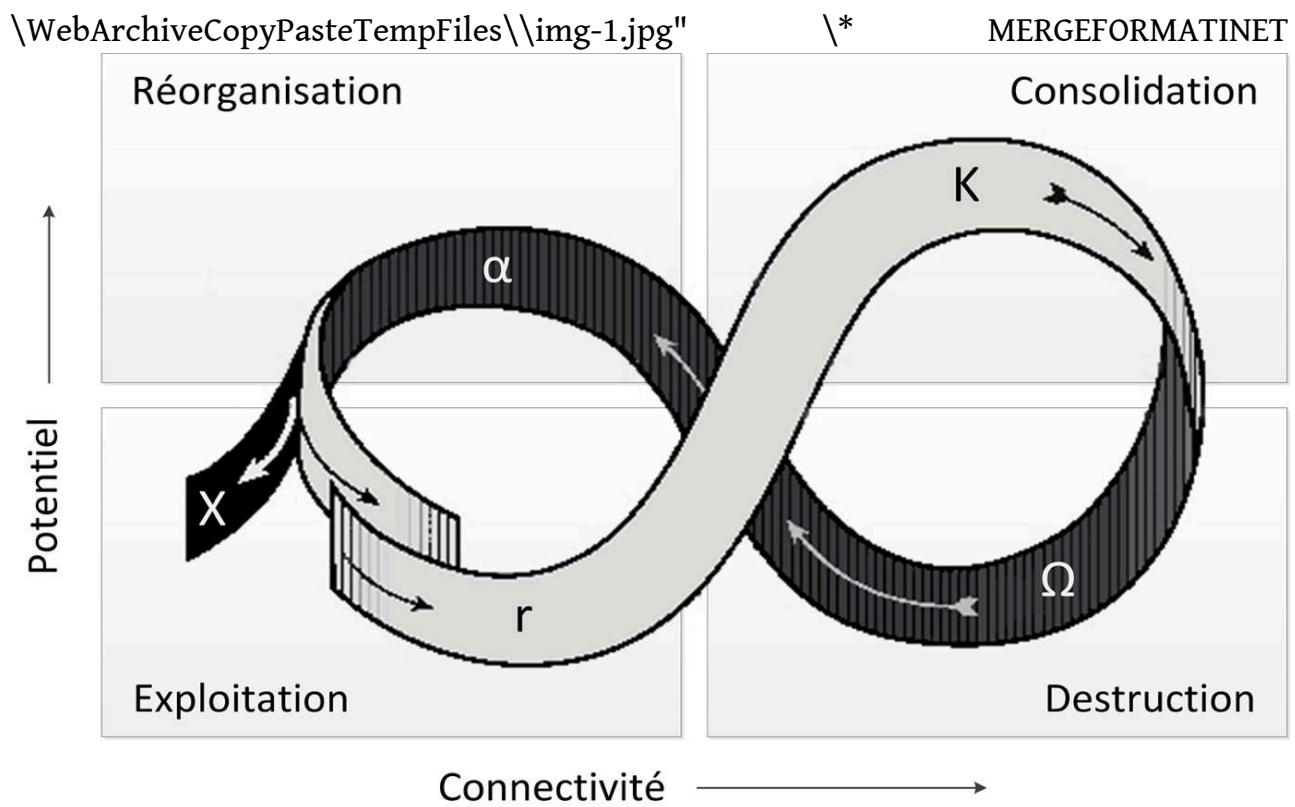

Gunderson, Holling, 2002

\section{Question de recherche et méthodologie}

7 Le choix de la Bosnie-Herzégovine comme cas d'étude de cette recherche vient souligner plusieurs lacunes. Plusieurs chercheurs se sont attardés sur les conséquences $\mathrm{du}$ conflit dans l'urbanité et sa reconstruction ${ }^{26}$, les représentations patrimoniales du conflit $^{27}$, l'impact sur les communautés et la société tri-ethnique ${ }^{28}$, la résilience postconflit allant, au niveau urbanistique, jusqu'à la prévention du risque en passant par le traumatisme psychologique ${ }^{29}$. Cependant, l'analyse de la résilience de la destination touristique avec pour catalyseur les nouvelles technologies n'a jusqu'à présent pas fait l'objet de recherches spécifiques concernant la Bosnie-Herzégovine, ni les Balkans plus largement, dans la littérature scientifique francophone. Comme le soulignent Cholat et al.,

les mutations contemporaines, passées ou futures des sociétés, les transformations des rapports de l'homme à l'espace et au temps, les nouvelles exigences des populations, la montée des incertitudes questionnent l'activité touristique et ses «adaptations» au sens général de «se conformer aux circonstances, se mettre en accord $»^{30}$.

Notre question de recherche va se structurer, de ce fait, autour de la place des initiatives technologiques et numériques dans la reconstruction et la résilience touristique de la Bosnie-Herzégovine post-conflit et de leur positionnement dans le processus de résilience adaptatif de Gunderson et Holling.

9 Pour éclairer cette question, nous avons procédé à une analyse documentaire des publications et rapports produits par le gouvernement de la fédération de BosnieHerzégovine, des articles scientifiques, des monographies et des articles de journaux portant sur la relance du tourisme bosnien après la guerre de 1992-1995, et nous avons également réalisé un séjour en Bosnie-Herzégovine, à Sarajevo et Mostar. Par la suite, nous avons tenté d'identifier les initiatives technologiques et numériques, les acteurs impliqués dans les processus de développement touristique, leurs rôles, les choix 
spécifiques de ces projets et leur apport potentiel à la résilience de la destination touristique bosnienne.

\section{Les technologies, au cœur de la reconstruction touristique territoriale}

10 Depuis le déploiement et l'explosion de la bulle internet à la fin du $\mathrm{xx}^{\mathrm{e}}$ siècle, le développement des technologies de l'information et de la communication (TIC) a entraîné des changements organisationnels profonds et une redéfinition importante des marchés dans l'activité touristique ${ }^{31}$. Le monde du tourisme est entré de plain-pied dans cette nouvelle révolution digitale et technologique. Pour Perrain et Jean-Pierre ${ }^{32}$, on passe de la forme dite de l'e-tourisme selon une configuration web 2.0, où l'internaute devient un actif, au modèle du tourisme intelligent, c'est-à-dire au web 3.0, celui de la mobilité, des objets connectés, des données, de l'intelligence artificielle et des robots. Les technologies de l'information étant de plus en plus intégrées dans les organisations et entités, les destinations touristiques exploitent de plus en plus les innovations technologiques pour soutenir l'enrichissement des expériences touristiques et le développement de la destination ${ }^{33}$. Conscient du potentiel technologique pour le développement touristique de la destination Bosnie, le gouvernement bosnien, en collaboration avec des acteurs du tourisme, de la culture et de l'ingénierie, priorise les investissements en faveur du développement des secteurs du numérique et des nouvelles technologies ${ }^{34}$.

11 L'importance des médias numériques et des nouvelles technologies dans le secteur touristique s'est affirmée depuis le début des années 2000. Elle prend forme non seulement dans la construction, la promotion, la diffusion de l'offre touristique, mais également dans la narration et la mise en discussion des expériences touristiques vécues $^{35}$. Les nouvelles technologies peuvent positivement contribuer à l'évolution des imaginaires, des attentes et des pratiques touristiques des visiteurs. La diffusion des images et des représentations touristiques n'a jamais paru aussi facile avec Internet. Les imaginaires émanant d'une destination alimentent le désir des touristes d'y aller ${ }^{36}$. Cependant, l'usage qui est fait de ces nouvelles technologies peut impacter négativement la destination en participant à la création d'un imaginaire parmi les visiteurs étrangers ${ }^{37}$. Ceci est particulièrement vrai dans le cas de la BosnieHerzégovine, confrontée à certaines pratiques (mémorielles, muséales, touristiques, artistiques) et à une nouvelle interprétation ou réinterprétation des territoires ${ }^{38}$.

Les nouvelles révolutions technologiques et digitales, dans le cas de destinations comme la Bosnie-Herzégovine, peuvent apporter une contribution à la reconstruction et à la résilience de la destination touristique, en valorisant les atouts existant avant le conflit (histoire, patrimoine culturel et naturel) et les nouveaux attraits (mémoire, innovation). En misant sur ce créneau technologique, le pays reconstruit son territoire touristique et déploie l'image d'une destination touristique renouvelée et intelligente ${ }^{39}$ qui va au-delà de la ville meurtrie et traumatique figée dans l'inconscient collectif ${ }^{40}$. L'un des projets les plus emblématiques de ce virage numérique est le projet « Sarajevo - Smart City Initiative 2020 », dont le but est de faire de Sarajevo une destination touristique régionale, leader dans les nouvelles technologies et l'intelligence artificielle, un City Mind Lab qui devrait piloter le processus et contribuer avec des idées et des solutions innovantes ${ }^{41}$. Ces dernières années, le concept de destinations touristiques 
intelligentes (Smart Tourism Destinations, STD) a émergé comme émanation du développement des villes intelligentes, ou smart cities. L'avènement des nouvelles technologies a entraîné l'émergence de villes intelligentes visant à fournir à leurs parties prenantes des solutions technologiques efficaces et efficientes. L'objectif des villes intelligentes est d'améliorer les résultats liés aux personnes, aux systèmes et aux processus des entreprises, du gouvernement et d'autres entités des secteurs public et privé. Leur objectif principal est d'améliorer la qualité de vie de tous les résidents ${ }^{42}$. Le concept de tourisme intelligent et numérique apparaît de plus en plus comme une composante du concept de ville intelligente, visant à fournir aux touristes des solutions répondant aux besoins spécifiques liés aux voyages ${ }^{43}$.

\section{Le numérique au service de la mémoire et de l'expérientiel ? « The Sarajevo Survival Tools Virtual Environment »}

13 Le projet «Sarajevo Survival Tools » est le fruit d'une collaboration entre le musée d'histoire de Bosnie-Herzégovine à Sarajevo et des étudiants de troisième année du département d'informatique de la faculté de génie électrique de Sarajevo, spécialisés dans le domaine de la numérisation du patrimoine culturel ${ }^{44}$. Dans ce projet, ne présentation virtuelle de l'exposition Sarajevo Under Siege du musée historique a ainsi été créée, rassemblant des objets créés et utilisés par les citoyens de Sarajevo pendant la période de trois ans et demi pendant laquelle la ville a été sous siège (1992-1996) ${ }^{45}$. Le concept de visites virtuelles muséales a émergé en même temps que l'explosion d'Internet dans les années 1990. Les musées virtuels sur Internet sont en expansion depuis une vingtaine d'années, visant à mettre en place une extension numérique du musée physique sur Internet ${ }^{46}$. Pour Terrisse ${ }^{47}$, même si l'on ne peut remplacer l'expérience et la découverte procurées par les visites réelles, les objectifs de ces premiers systèmes virtuels se focalisent sur une diffusion d'œuvres numérisées à l'intention des spécialistes et du grand public. Les buts poursuivis sont à la fois éducatifs, scientifiques et touristiques, notamment dans le cas des publics éloignés. À la différence de ce que décrit Glassner ${ }^{48}$, qui définit la narration interactive comme étant une expérience à double sens, au cours de laquelle le visiteur qui se trouve dans l'auditoire affecte réellement l'histoire elle-même, le projet " Sarajevo Survival Tools » permet aux utilisateurs d'approfondir leurs connaissances et leur expérience de l'évènement historique. L'utilisateur ne modifie pas l'intrigue de l'histoire, mais il élargit son expérience en visualisant les présentations interactives des objets mentionnés dans l'histoire ${ }^{49}$. 
Figure 2. Page d'accueil du site « Sarajevo Survival Tools Virtual Environment »

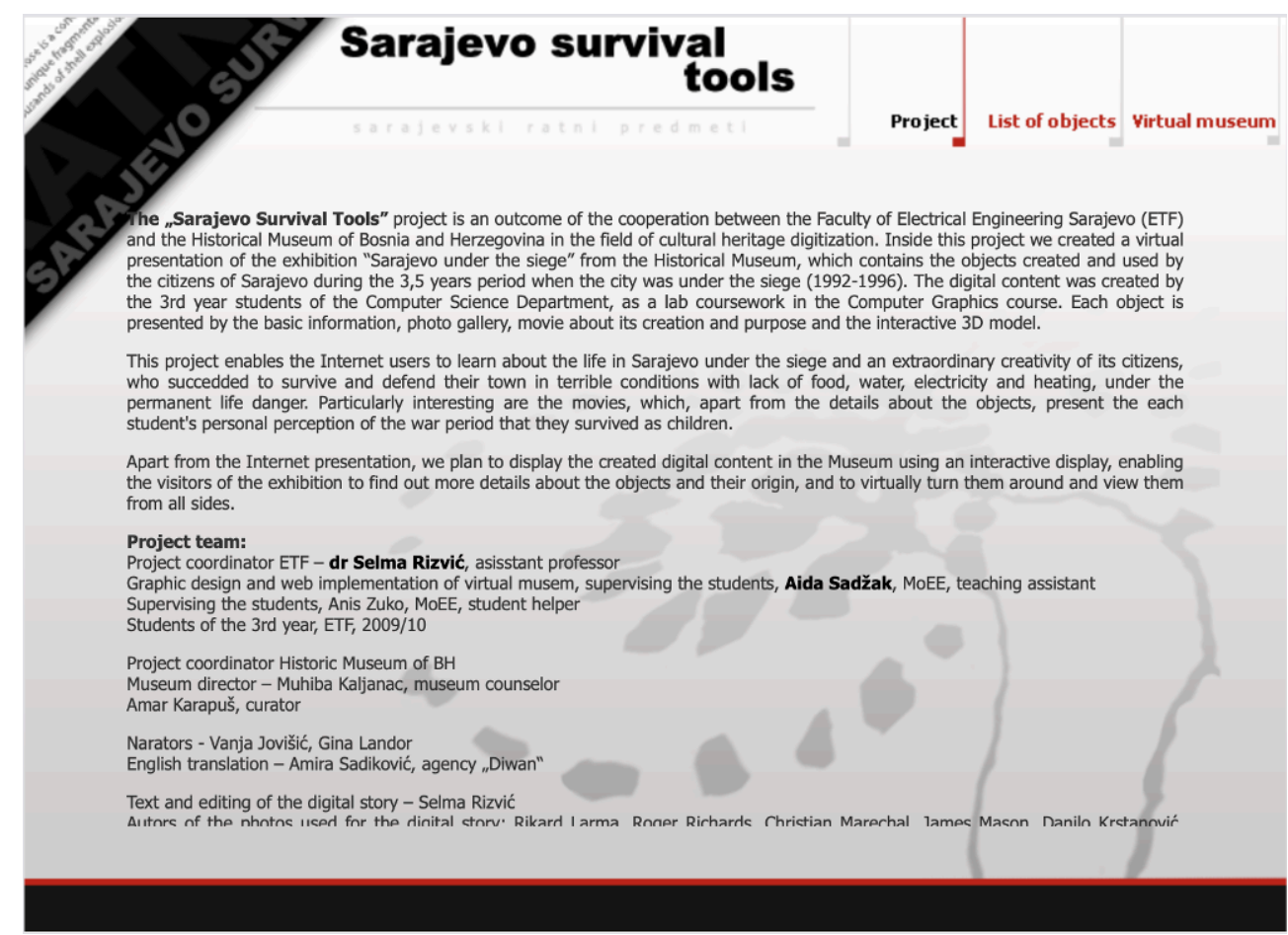

Sarajevo Survival Tools, 2020

Comme indiqué dans la navigation du site, le but de cet outil est d'offrir un premier aperçu aux visiteurs de la réalité quotidienne des habitants de Sarajevo durant le siège (1992-1995). Les options de présentation d'objets du quotidien durant le conflit et de visites virtuelles sont avant tout une incitation à visiter le musée d'histoire de la Bosnie-Herzégovine de Sarajevo. Si l'utilisateur visite le musée plus d'une fois, il ne voudra plus revoir les mêmes segments d'histoire et devrait avoir la possibilité de les ignorer et de naviguer directement vers des groupes d'objets particuliers ${ }^{50}$. Également présentes sur le site, des vidéos documentent la perception personnelle de chacun des élèves impliqués dans le projet "Sarajevo Survival Tools Virtual Environment", décrivant leurs souvenirs de la guerre dans leur enfance. Au-delà du musée d'histoire de la Bosnie-Herzégovine, beaucoup de musées à travers le monde ont recours à la réalité virtuelle, tels le Smithsonian Museum de Washington avec son «Smithsonian virtual tour ", le musée Van Gogh d'Amsterdam et son "Van Gogh virtual tour » ou encore le musée virtuel 3D des Inuits au Canada ${ }^{51}$. Pour Schweibenz ${ }^{52}$, ces musées virtuels ont pour objectif de prolonger les différentes idées et concepts des collections dans le cyberespace tout en révélant la quintessence du musée. Parallèlement, le musée virtuel touche des visiteurs qui n'auront peut-être jamais la possibilité de se rendre physiquement dans l'un de ces musées. 
Figure 3. Exemple d'objets présentés dans le « Sarajevo Survival Tools Virtual Environment »

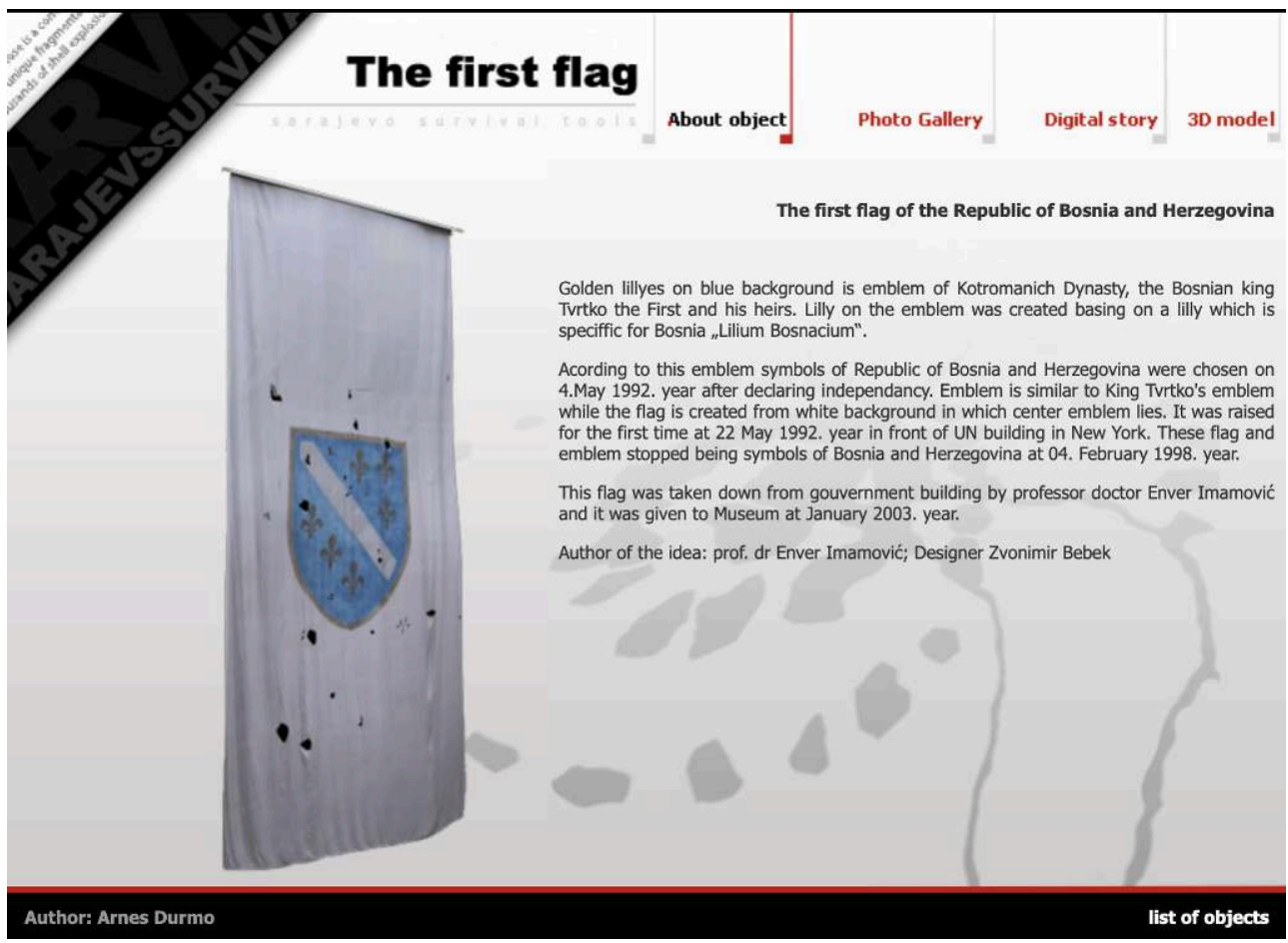

Sarajevo Survival Tools, 2020

Si ce projet, réalisé il y a dix ans maintenant, a été construit à des fins louables de promotion du musée d'histoire de Bosnie-Herzégovine de Sarajevo, les défauts sont récurrents. Depuis 2020, le site n'a plus été modifié ou mis à jour par ses créateurs ni par des employés du musée. L'obsolescence des graphismes et l'impossibilité de faire la visite virtuelle à partir d'un ordinateur de type mac ou une tablette numérique nuisent à toute tentative de découverte par les visiteurs. Cet « abandon » du site est regrettable, alors que l'élaboration d'un espace virtuel comme «Sarajevo Survival Tools » a pour vocation première d'offrir un sentiment d'implication et d'interactivité plus fort entre les visiteurs et le musée. La recherche de Risvic souligne ce succès auprès d'un panel de 46 personnes âgées de 22 à 65 ans, avec un âge moyen de 33 ans, qui se sont portées volontaires pour l'expérience. Le panel a attribué la note de 4,35/5 à la pertinence de l'histoire numérique durant la visite virtuelle pour la transmission de l'information et la note de 3,64/5 à la motivation à découvrir plus d'objets liés au conflit à travers l'histoire numérique ${ }^{53}$. Ces éléments de notation montrent l'intérêt et le succès des visites virtuelles du musée d'histoire de Bosnie-Herzégovine dans une perspective de (re)découverte de la vie à Sarajevo pendant le conflit.

La thématique du siège de Sarajevo interroge l'interprétation du conflit et la neutralité $\mathrm{du}$ regard offert au visiteur dans un contexte de frictions ethniques récurrentes. Si l'existence d'un musée virtuel a pour mission de favoriser l'intérêt pour l'exploration réelle du musée et la visite d'un public large, les acteurs culturels de la capitale bosnienne se retrouvent en porte-à-faux avec les acteurs politiques locaux, surtout fédéraux, qui n'ont pas la même lecture du conflit ${ }^{54}$. La guerre en Bosnie a profondément transformé la perception de la culture et le rôle des musées en BosnieHerzégovine. La question identitaire affecte les représentations historiques triethniques dans les musées bosniens de la capitale, fondés en majeure partie durant 
l'ère multiculturelle yougoslave. Politiquement, les musées sarajéviens sont gérés au niveau étatique, se plaçant en dehors de la juridiction des deux entités fédérales, qui ne parviennent pas à se mettre d'accord sur une même ligne directrice, nourrissant la fracture identitaire ${ }^{55}$. La culture nationale tombe dans un vide institutionnel. Il n'existe pas de ligne de budget dédiée à la culture. Le musée d'histoire reçoit seulement des subventions du ministère des Affaires civiles ${ }^{56}$. Suite aux accords de Dayton, le statut des instituts culturels n'a jamais été actualisé et aucun ministère de la Culture n'a vu le jour. Avec un budget minime de 1,5 million d'euros annuels, à répartir entre ses huit institutions culturelles ${ }^{57}$, l'État ne peut plus assurer leur survie :

Renforcer le budget et les prérogatives de l'État dans le domaine culturel entrâne le refus des partis nationalistes, comme le SNSD (Alliance indépendante des sociaux-démocrates, parti nationaliste serbe de Milorad Dodik, dirigeant de la Republika Srpska), pas disposé à prendre en charge des institutions exemptes de connotations nationales, refusant ainsi l'idée même de l'existence d'un patrimoine culturel commun. Plus qu'un simple problème financier, le réel nerf de la guerre semble surtout politique, lié à la reconnaissance ou non d'un patrimoine commun. ${ }^{58}$

Figure 4. Exposition permanente sur le siège de Sarajevo, musée d'histoire de la BosnieHerzégovine

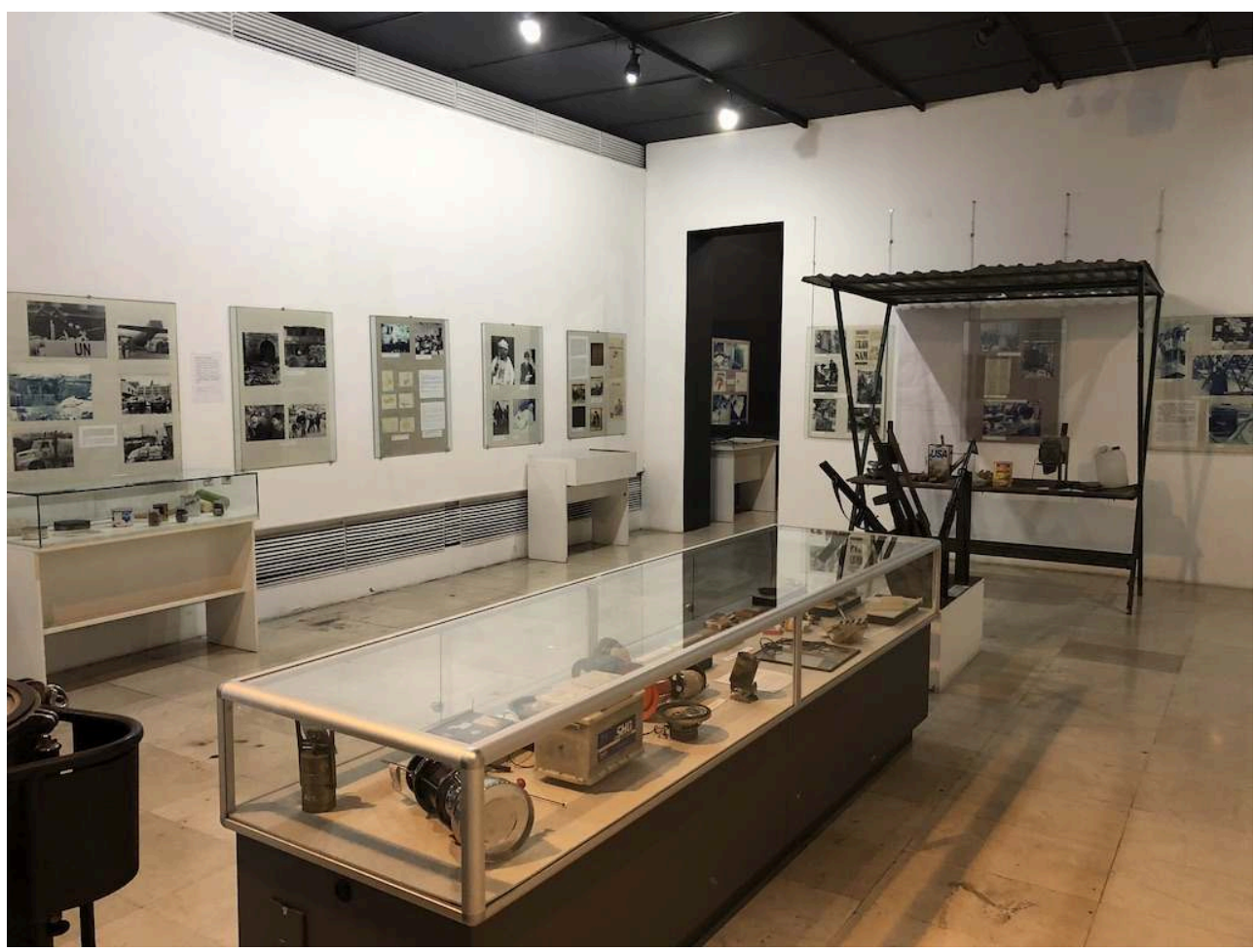

Photo de l'auteur, 2019

17 Ce manque de crédits budgétaires, causé par le système politique hautement décentralisé et les frictions entre représentants des communautés ethniques ${ }^{59}$, défavorise le développement de projets innovants locaux, ainsi que la mise en patrimoine de la mémoire du conflit selon un consensus entre les populations constitutives $^{60}$. Familiariser les visiteurs et les populations locales avec le conflit passé rentre dans une logique de résilience territoriale et des populations par la culture. Dans le cas du musée d'histoire de Sarajevo, le rôle joué par la culture dans le développement d'une résilience urbaine durable dépasse sa simple valeur de bien ou de ressource 
permettant d'attirer des investissements et de renforcer une image de marque. La vitalité culturelle est essentielle à la vie urbaine et sociétale, car elle imprègne tous les aspects de l'existence et est au fondement même des libertés, de l'échange d'idées et du bien-être sociétal ${ }^{61}$. L'utilisation de la réalité virtuelle, au-delà d'attirer les touristes étrangers, doit favoriser le processus post-conflit et de résilience des communautés victimes du siège de Sarajevo par la mémorialisation du conflit et de ses représentations ${ }^{62}$, en collaboration avec des acteurs de chaque ethnie constitutive. Volfgang Petritsch, ancien Haut-représentant pour la Bosnie-Herzégovine de 1999 à 2002, définit trois approches différentes dans la mise sur pied d'un processus de réconciliation : « la première liée à la justice transitionnelle - tribunaux internationaux et locaux -, la deuxième attachée à la reconstruction économique et finalement l'approche culturelle, incluant les commémorations, les musées, les films, etc. ${ }^{63}{ }$. Ces approches se veulent fondées sur une participation pleine et active des habitants. Le manque de crédits budgétaires et de moyens pour entretenir le musée virtuel, dix ans après sa création, est à l'image du musée physique lui-même, en sommeil. Certaines pratiques (mémorielles, muséales, touristiques, artistiques) participent à la mise en mémoire du conflit, à travers la patrimonialisation d'objets issus du siège de la ville ${ }^{64}$. Cette initiative de musées virtuels, de par leurs succès un peu partout à travers le monde, serait pour les musées sarajéviens et bosniens un modèle d'attractivité touristique et de pédagogie culturelle sur le conflit passé.

\section{La smart city comme modèle d'intégration et d'attractivité touristique ? Le projet « Sarajevo Smart City Hackathon 2020 »}

18 Washburn et Sindhu définissent la ville intelligente (smart city) comme :

une utilisation combinée de systèmes logiciels, d'infrastructures de serveurs, d'infrastructures de réseau et de périphériques clients - que Forrester appelle les technologies de calcul intelligent - pour mieux connecter sept composants et services essentiels de l'infrastructure de la ville: administration de la ville, éducation, santé, sécurité publique, immobilier, transports et utilitaires ${ }^{65}$.

La croissance rapide de la population mondiale et urbaine pose de nouveaux défis pour les services et les infrastructures des villes. Le concept de smart city propose de nouvelles opportunités économiques et sociales pour les populations. Elle promet notamment de capitaliser sur ses opportunités économiques et ses avantages sociaux tout en allégeant les souffrances liées à l'urbanisation ${ }^{66}$.

Conscients de ces nouveaux défis et du besoin d'attirer de nouveaux investissements, de jeunes entrepreneurs et ingénieurs de toute la Bosnie-Herzégovine ont lancé en 2015 le concept "Smart Sarajevo ", afin d'attirer les grands noms de la Tech et de la Silicon Valley (IBM, Microsoft, Apple, etc.) et de positionner le pays en tant que hub d'innovation et de nouvelles technologies de portée régionale. En 2018, la ville de Sarajevo et le Programme des Nations unies pour le développement (PNUD) ont lancé le projet "Sarajevo smart city Hackathon 2020 ». La première étape de ce projet est la création d'une zone pilote dans la ville de Sarajevo, choisie comme «terrain d'essai » pour la mise en œuvre de plusieurs activités interconnectées. Le projet facilitera la conception d'un outil de réalité virtuelle/3D d'aide à la visualisation territoriale et la création de la projection "mahala numérique 2050 », le mot mahala (sing.) désignant les quartiers du vieux centre-ville ottoman de Sarajevo. Ce projet, basé sur une vision 
citoyenne, soutiendra des initiatives à petite échelle qui aideront à transformer la vision en réalité :

Le développement de la ville de Sarajevo s'accélère en termes d'infrastructure, d'économie, de croissance du tourisme et la ville devient un centre régional. Cela doit s'accompagner de l'application de solutions contemporaines et intelligentes par l'administration municipale. L'introduction des technologies intelligentes est considérée comme l'une des priorités de cette administration, c'est pourquoi l'initiative que nous lançons aujourd'hui représente un pas important dans la bonne direction ${ }^{67}$.

Figure 5. Logo du projet « Sarajevo smart city Hackathon 2020 »

INCLUDEPICTURE "..././../../var/folders/9g/z6yww88s1nlbbyb0wf0w06vw0000gn/T/ com.microsoft.Word/WebArchiveCopyPasteTempFiles/sarajevo-pametna-

buducnost45.jpg\%3fzoom=2\&resize=780,298\&ssl=1" $\quad \backslash^{*} \quad$ MERGEFORMAT

INCLUDEPICTURE $\quad$ "/Users/../../../../var/folders/9g/

z6yww88s1nlbbyb0wf0w06vw0000gn/T/com.microsoft.Word/

WebArchiveCopyPasteTempFiles/sarajevo-pametna-buducnost45.jpg?

zoom=2\&resize=780,298\&ssl=1" \* MERGEFORMATINET INCLUDEPICTURE "\\\|Users \}

$\backslash . . \backslash . . \backslash$.. $\backslash \backslash . . \backslash$ var $\backslash \backslash$ folders $\backslash \backslash 9 g \backslash \backslash z 6 y w w 88 s 1$ nlbbyb0wf0w06vw0000gn $\backslash \backslash T \backslash$

\com.microsoft.Word $\backslash \backslash$ WebArchiveCopyPasteTempFiles $\backslash \backslash$ sarajevo-pametna-

buducnost45.jpg?zoom=2\&resize $=780,298 \&$ ssl $=1 " \quad \backslash^{*} \quad$ MERGEFORMATINET

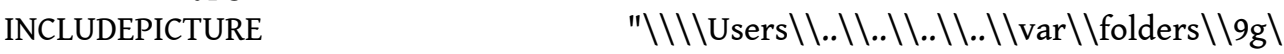

$\backslash$ z6yww88s1nlbbyb0wf0w06vw0000gn $\backslash \backslash T \backslash \backslash$ com.microsoft.Word $\backslash$

$\backslash$ WebArchiveCopyPasteTempFiles \\sarajevo-pametna-buducnost45.jpg?

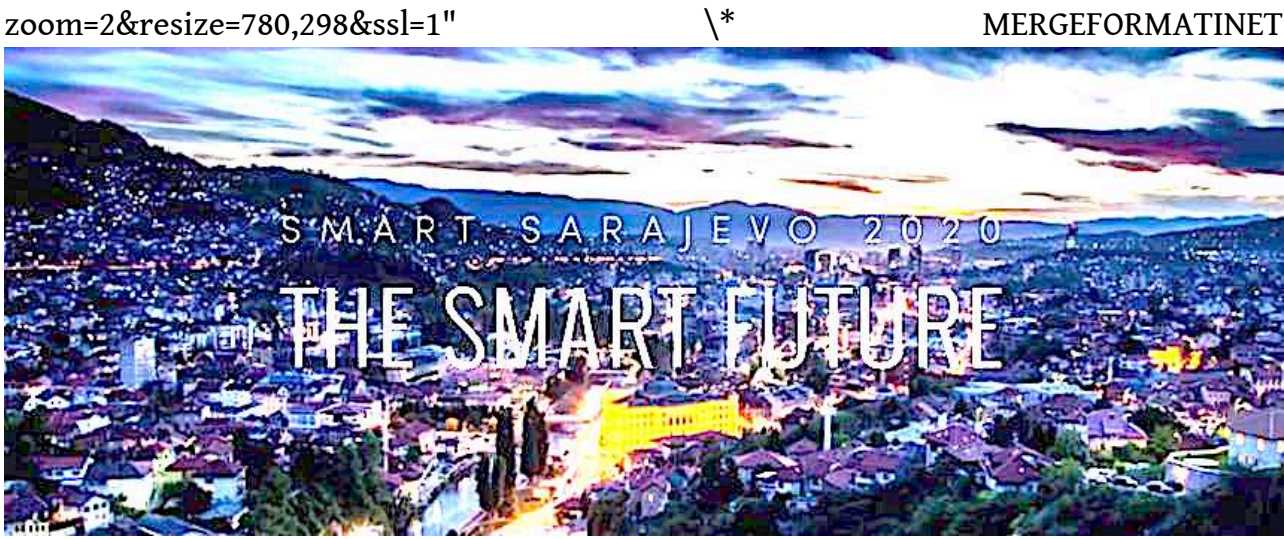

City OS Hackathon, 2015

21 Le secteur touristique est partie prenante dans le développement du projet «Sarajevo smart city ». L'un des derniers exemples en date est l'installation de structures écointelligentes pour le tourisme de santé, s'adressant à des visiteurs de 65 ans et plus, retraités ${ }^{68}$, avec pour objectif de mettre en place des initiatives et des solutions visant la santé, la relaxation et le bien-être en milieu urbain et naturel :

l'introduction de structures d'hébergement pour personnes âgées basées sur des solutions éco-intelligentes avec un accent mis sur le bien-être, la santé, le mode de vie actif et les voyages. L'objectif est d'approfondir la compréhension du tourisme des seniors en tant que concept relativement nouveau du tourisme et d'explorer les opportunités d'ouverture d'une nouvelle installation touristique dans la région de Sarajevo sous la forme d'une étude de faisabilité et d'un plan stratégique spécialement conçu pour les touristes seniors ${ }^{69}$. 
De par ses nombreux attraits (environnement naturel riche en montagnes et forêts, gastronomie variée et multiculturelle, patrimoine historique et culturel), Sarajevo et sa région possèdent un potentiel élevé de destination de tourisme curatif et récréatif pour des visiteurs en forte demande de voyage, de découvertes et de dépaysement ${ }^{70}$. Pour développer ce potentiel touristique, de nouveaux projets d'infrastructures doivent voir le jour.

Figure 6. Signature du projet « Sarajevo Smart City » entre le maire de Sarajevo, Abdulah Skaka, et le représentant du PNUD, Sukhrob Khoshmukhamedov

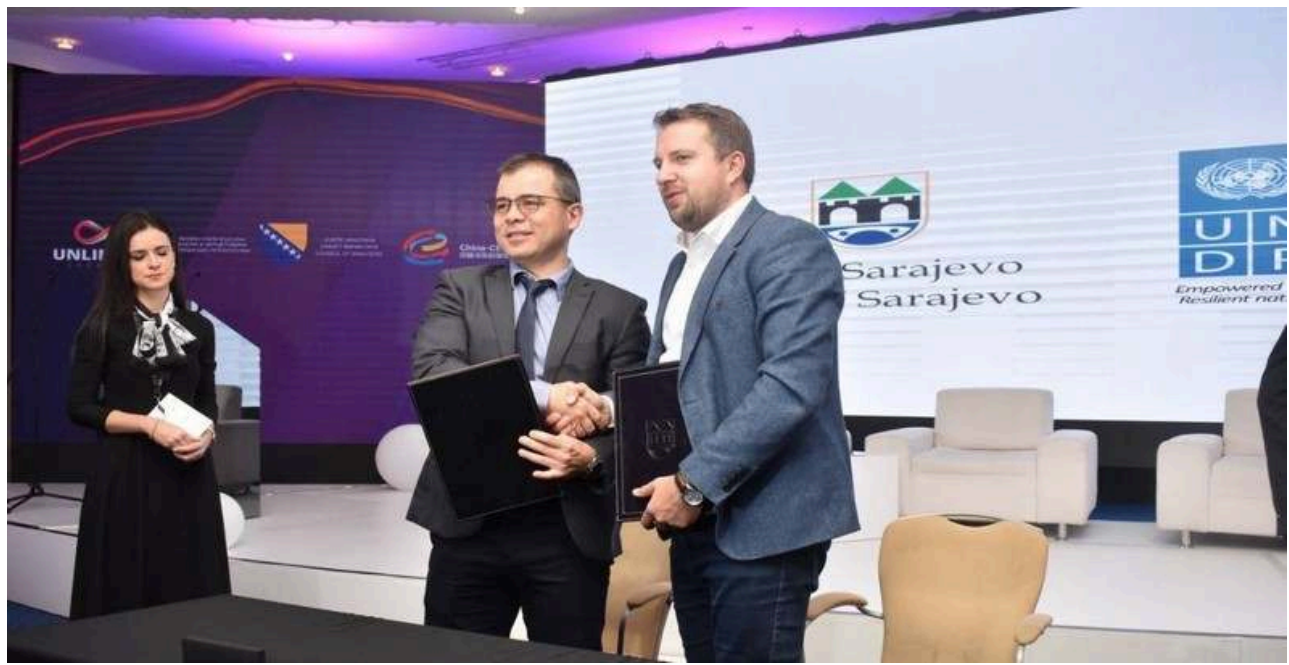

PNUD, 2018

La smart city est aujourd'hui un outil d'intelligence territoriale en plein essor qui représente une opportunité de répondre aux multiples enjeux (environnementaux, sociaux et économiques) des villes. En 2050 , près de $70 \%$ de la population mondiale vivra en ville. Si l'avancée des nouvelles technologies et l'exploitation des données massives permettent de créer des synergies répondant aux besoins de toutes les parties prenantes des territoires, la smart city peut également être perçue comme un instrument de soft power tant le développement durable fait l'objet d'une problématique internationale ${ }^{71}$. Le projet smart city à Sarajevo tend vers une approche de résilience adaptative de la destination touristique. L'adaptativité de la résilience se concentre principalement sur les processus de destruction et de réorganisation territoriale, souvent négligés en faveur de la croissance économique et de la conservation des acquis. Pour Gunderson et Holling ${ }^{72}$, l'inclusion de ces processus offre une vision plus complète de la complexité de la dynamique du système urbain qui relie l'organisation du système, la résilience et la dynamique. Un cycle adaptatif alternant entre de longues périodes d'agrégation et de transformation des ressources et des périodes plus courtes qui créent des opportunités d'innovation est proposé comme unité fondamentale pour comprendre les systèmes complexes des cellules aux écosystèmes en passant par les sociétés ${ }^{73}$. Tourné vers une vision à long terme, le développement de Sarajevo en tant que ville intelligente doit prendre en compte les incertitudes globales du système urbain et de sa gouvernance, quant aux les évolutions de l'environnement physique, technologique, économique, social et gouvernemental. Les parties prenantes du projet "Smart City Sarajevo" font le choix d'une série de projets et d'actions localisés à l'échelle micro pour pallier les vulnérabilités internes et externes des 
dysfonctionnements et fractures à l'échelle urbaine et territoriale, afin d'anticiper l'adaptation du fonctionnement du système urbain et de ses multiples composants ${ }^{74}$.

\section{Conclusion}

24 Les politiques de développement économique dans le tourisme en Bosnie-Herzégovine occupent une place de plus en plus importante en tant que facteur d'activités de développement et de restructuration. Le tourisme est multisectoriel, il touche de nombreuses franges de la société et de l'économie (hôtellerie, gastronomie, transports, environnement, industrie ou encore éducation). Le tourisme contribue globalement au développement des pays en rehaussant leur prospérité et leur niveau de vie, ce qui favorise et soutient le développement touristique. Conscients de ces enjeux, les acteurs politiques, institutionnels et économiques bosniens misent sur le développement de nouvelles ressources technologiques et numériques pour toucher de plus grands segments de visiteurs domestiques et étrangers (histoire, patrimoine urbain et naturel, santé, thermalisme, etc.) $)^{75}$. Plusieurs projets à différentes échelles se développent ou sont en cours d'élaboration, non seulement pour faire de la Bosnie-Herzégovine une destination touristique de rang européen et mondial, mais aussi afin de casser l'image récurrente de la guerre et du conflit ethnique, toujours présente dans les mémoires. Les nouvelles technologies dans le tourisme ont un rôle à jouer dans le développement de la destination Bosnie-Herzégovine. Si, pour l'heure, nous ne pouvons confirmer un tournant numérique du tourisme bosnien, un élan est néanmoins visible dans la planification touristique du pays. Les plus grands défis pour le pays résident dans le complexe processus de transition post-conflit à l'échelle politique qui, en dépit d'efforts aux différents échelons décisionnels, crée des entraves en lien avec l'héritage du conflit sur son système politique et constitutionnel, parmi les plus complexes et inopérants au monde ${ }^{76}$. Si la précarité institutionnelle est toujours évidente à l'heure actuelle, la forte déconcentration des pouvoirs étatiques vers le local permet néanmoins l'émergence de projets dans les secteurs économiques de la capitale et du pays ${ }^{77}$.

Cependant, un élan touristique se dessine via la multiplication des projets en faveur de la reconstruction de la destination Bosnie-Herzégovine. Le pays et son territoire doivent être perçus par les acteurs de l'industrie touristique comme des systèmes socio-écologiques complexes, sujets à des cycles adaptatifs permanents comprenant un effondrement ${ }^{78}$ et présentant donc une tendance inhérente à la crise ${ }^{79}$. La diversité culturelle des populations constitutives de la Bosnie-Herzégovine, le traumatisme latent du conflit et la difficulté à gouverner et se mettre d'accord entre les différents acteurs du pays, extrêmement périclités dans leurs intérêts ethniques et historiques respectifs, complexifient au maximum l'élaboration d'un processus de résilience du territoire, glissant davantage vers les initiatives locales et citoyennes. L'usage des nouvelles technologies et des initiatives locales apparaît comme une réponse prometteuse aux projets de reconstruction de la destination touristique et de reconstruction de son image dans une globalité à l'échelle du pays.

Les prochaines années vont s'avérer cruciales pour le pays et la région. Entre 2002 et 2012, la fréquentation touristique a évolué en dents de scie et le Forum économique mondial a classé en 2017 la Bosnie Herzégovine au $113^{\mathrm{e}}$ rang sur 134 en termes de compétitivité touristique ${ }^{80}$. Néanmoins, le pays a affiché le troisième taux de croissance touristique le plus élevé au monde sur la période $1995-2020^{81}$ et le nombre d'arrivées de 
touristes internationaux devrait passer de 700000 en 2017 à 950000 en 2018, soit un record de fréquentation dans l'industrie touristique bosnienne ${ }^{82}$. La pandémie de Covid-19, toujours d'actualité à l'heure où nous écrivons, va ajouter de l'instabilité et de nouveaux défis pour le secteur touristique pour l'atteinte des ambitieux objectifs gouvernementaux.

La place du numérique et des nouvelles technologies dans l'industrie touristique du pays devient un facteur de plus en plus déterminant dans la reconstruction et la résilience touristique à venir. Devenues incontournables dans un grand nombre de destinations touristiques dans le monde, les nouvelles technologies de l'information, au travers d'initiatives locales présentées plus haut, offrent un aperçu des possibilités inhérentes pour le développement et l'attractivité touristique globale d'un pays ayant connu un conflit majeur il y a presque trente ans. Ces projets mettent l'emphase sur la richesse et le potentiel touristique toujours existant, de par son historicité et qui se reconstruit année après année. Si la difficile collaboration entre acteurs fédéraux et étatiques et les tensions interethniques ralentissent, au niveau global, les décisions, sous fond de fracture et de partition identitaires, les initiatives locales entre populations, chercheurs et acteurs locaux et associatifs nourrissent la résilience communautaire. Cette résilience des communautés contribue au succès croissant des projets mis en place, transformant l'image rémanente d'un pays en guerre en une destination touristique offrant une variété d'atouts, facteurs de nouvelles représentations et d'images, diffusées aux touristes. Le tourisme défini comme intelligent dans le cas de la Bosnie-Herzégovine s'inscrit dans une logique transversale de reconstruction et de résilience de la destination touristique en termes d'infrastructures, de représentations touristiques et d'attractivités multiples. Si les défis existants étaient déjà nombreux, notamment eu égard au système politique en place, aux infrastructures vieillissantes et à la situation économique, la pandémie mondiale de Covid-19 va forcer les acteurs touristiques à trouver de nouvelles solutions innovantes pour propulser la destination bosnienne au rang de destination touristique majeure. Les prochaines années vont s'avérer cruciales pour la mise en œuvre de nouveaux projets touristiques et l'intensification des technologies, afin d'accomplir la reconstruction de la destination et élaborer diverses stratégies aux différents niveaux (local, fédéral, national) et paliers (populations locales, acteurs institutionnels, académiques, touristiques), permettant la résilience post-conflit et post-Covid du secteur touristique bosnien et sa pérennisation.

\section{NOTES}

1. JEBRAK Yona, La reconstruction et la résilience urbaine: l'évolution du paysage urbain, thèse de doctorat en études urbaines, Montréal, Université du Québec à Montréal, 2010.

2. DETRY Nicolas, Le patrimoine martyr et la restauration post bellica en Europe, thèse de doctorat en architecture, Lyon, École nationale supérieure d'architecture de Lyon, 2016.

3. BAUdour Rémi, « De la menace atomique aux "conflits de faible intensité" : l'emprise croissante de la guerre sur la ville », Les Annales de la recherche urbaine, $n^{\circ}$ 91, 2001, p. 27-34. 
4. DUBOIS-MAURY Jocelyne, «Les grandes métropoles face aux risques naturels et technologiques », Sécurité et stratégie, 2012, p. 47-55 ; STATHOPOULOs Marcos, «Qu'est-ce que la résilience urbaine?», Urbanisme, vol. 6, n 381, 2011, p. 90-92.

5. кномsi Mohamed Réda, "La résilience organisationnelle comme outil de relance d'une destination. Le cas du Québec », Études caribéennes, vol. 2, 2018, p. 1-15.

6. Ibid.

7. PERRAIN David, JEAN-PIERRE Philippe, La stratégie de destination intelligente, facteur clé des mutations des destinations touristiques vulnérables?, Saint-Denis de La Réunion, Université de La Réunion, 2019 p. 2.

8. BLAKE Adam, Sinclair Thea, SUGIYARTo Guntur, «Quantifying the Impact of Foot and Mouth Disease on Tourism and the UK Economy », Tourism Economics, vol. 33, nº 9, 2003, p. 449-465.

9. TCHOUKARINE Igor, «Un espace offert au tourisme : représentations de la Yougoslavie dans les guides touristiques imprimés français et yougoslaves au $\mathrm{xx}^{\mathrm{e}}$ siècle ", Études balkaniques, vol.12, 2005, p. 1-28 (8).

10. BALDO Martina, «Bosnia and Herzegovina, New Products and Investments to Boost the Tourism Industry», Twissen, 2019, en ligne: http://twissen.com/destinations/bosnia-andherzegovina-new-products-and-investments-to-boost-the-tourism-industry/ (consulté en septembre 2021).

11. PERRAIN, JEAN-PIERRE, La stratégie de destination intelligente, op. cit.

12. NAEF Patrick James, « Voyage à travers un baril de poudre : guerre et imaginaire touristique à Sarajevo » Via, vol. 1, 2012, p. 1-10.

13. NAEF Patrick James, "Tourisme de mémoire, instrument de paix et/ou de réconciliation", Quand le tourisme questionne la mémoire, Actes du colloque ASBL Tourisme autrement, 2013; KASSOUHA Zeid, «Tourisme(s) post-conflit : patrimoines, mémoires et interprétation, le temps de la résilience ", Séminaire "Patrimoines et mémoires post-conflit ", Lyon, Université de Lyon, 2019.

14. Ibid.

15. DELAPLACE Marie et al., «Risques, résilience et pérennité des destinations touristiques : une introduction ", Études caribéennes, $\mathrm{n}^{\circ}$ 2, 2018, p. 1-8.

16. Ibid.

17. HERNANDEZ Julie, «Le tourisme macabre à La Nouvelle-Orléans après Katrina : résilience et mémorialisation des espaces affectés par des catastrophes majeures », Norois, $n^{\circ} 208,2008$, p. 61-73.

18. URRY John, The Tourist Gaze, Londres, Routledge, 1990.

19. Ibid.

20. NAEF, « Voyage à travers un baril de poudre », art. cité ; KASSOUHA, « Tourisme(s) post-conflit », art. cité.

21. PELLING Marc, Natural Disasters and Development in a Globalizing World, Londres, Routledge, 2003, p. 100.

22. TOUBIN Marie et al., "La Résilience urbaine: un nouveau concept opérationnel vecteur de durabilité urbaine ?", Développement durable et territoires, vol. 3, $n^{\circ}$ 1, 2012, p. 1-19.

23. WALKER Brian, SALT David Andrew, Resilience Thinking, Washington, Island Press, 2006 ; HARVEY LEMELIN Raynald et al., « Résilience, appartenance et tourisme à Nain », Études/Inuit/Studies, vol. 36, $\mathrm{n}^{\circ}$ 2, 2012, p. 35-58.

24. GUNDERSON Lance, HOLLING Crawford Stanley, (dir.), Panarchy: Understanding Transformations in Human and Natural Systems, Washington D.C., Island Press, 2002.

25. cholat Florent et al., Tourisme(s) et adaptation(s), Grenoble, Elya, 2019.

26. AQUILUÉ Inés, ROCA Estanislao, « Urban Development after the Bosnian War: The Division of Sarajevo's Territory and the Construction of East Sarajevo ", Cities, vol. 58, 2016, p. 152-163. 
27. NAEF, « Voyage à travers un baril de poudre ", art. cité ; KASSOUHA, « Tourisme(s) post-conflit », art. cité.

28. SANGUIN André Louis, « Retour à Srebrenica (Bosnie), notes de terrain à propos d'un nettoyage ethnique devenu génocide culturel », Géographie et cultures, n² 27, 1998, p. 63-78.

29. ALMEDOM Astier, «Social Capital and Mental Health: An Interdisciplinary Review of Primary Evidence », Social Science \& Medicine, vol. 61, n 5, 2005, p. 943-964.

30. CHOLAT et al., Tourismes et adaptations, op. cit., p. 30.

31. MARIANI Marcello et al., "Managing Change in Tourism Destinations: Key Issues and Current Trends », Journal of Destination Marketing \& Management, vol. 2, 2014, p. 269-272.

32. PERRAIN, JEAN-PIERRE, La stratégie de destination intelligente, op. cit.

33. BUHALIS Dimitrios, AMARANGgANA Aditya, "Smart Tourism Destinations: Enhancing Tourism Experience Through Personalisation of Services", dans Alessandro Inversini, Lia Tussyadiah (dir.), Information and Communication Technologies in Tourism, Berlin, Spinger, 2014, p. 377-389.

34. BALDO, « Bosnia and Herzegovina, New Products and Investments », art. cité.

35. PAGÉs Dominique, "Le tourisme créatif à la rencontre des cultures numériques: de la gouvernance des destinations à l'écriture des expériences ", Mondes du Tourisme, $\mathrm{n}^{\circ}$ 10, 2014, p. 75-90.

36. JAFARI Jafar, «Tourism Models: The Sociocultural Aspects ", Tourism Management, vol. $8, \mathrm{n}^{\circ} 2$, 1987, p. 151-159.

37. NAEF, « Voyage à travers un baril de poudre », art. cité.

38. KASSOUHA, « Tourisme(s) post-conflit », art. cité.

39. PERRAIN, JEAN-PIERRE, La stratégie de destination intelligente, op. cit.

40. NAEF, "Voyage à travers un baril de poudre ", art. cité ; NAEF, " Tourisme de mémoire ", art. cité ; TRATNJEK Bénédicte, «Des symboles dans la ville: Vučko, la mascotte des Jeux olympiques d'hiver de 1984 à Sarajevo ", Géographie de la ville en guerre, 2014, en ligne : http://geographieville-en-guerre.blogspot.com/2014/02/des-symboles-dans-la-ville-vucko-la.html (consulté en janvier 2019).

41. UNDP, "Sarajevo - Smart City Initiative" Launched", United Nations Development Programme (UNDP) in Bosnia and Herzegovina, 2018, en ligne: http://www.ba.undp.org/ content/bosnia_and_herzegovina/en/home/presscenter/articles/2018/_sarajevo-_-smart-cityinitiative-launched.html (consulté en septembre 2021).

42. KHAN Sajid, "Smart City and Smart Tourism: A Case of Dubai », Sustainability, vol. 9, $\mathrm{n}^{\circ} 12$, 2017, p. 1-24.

43. Ibid.

44. RISVIC Selma et al., «Interactive Digital Storytelling in the Sarajevo Survival Tools Virtual Environment ", SCCG'12: Proceedings of the $28^{\text {th }}$ Spring Conference on Computer Graphics, 2012, p. 109-116.

45. Ibid.

46. schWEIBENZ Werner, « Le musée virtuel », Les nouvelles de l'ICOM, $\mathrm{n}^{\circ}$ 3, 2004, p. 1-11.

47. TERRISSE Marc, «Musées et visites virtuelles : évolutions et possibilités de développement », Muséologies, vol. 6, $\mathrm{n}^{\circ}$ 2, 2013, p. 15-32.

48. GLASSNER Andrew, Interactive Storytelling, Natick Massachusetts, A. K. Peters, 2004.

49. RISVIC et al., « Interactive Digital Storytelling », art. cité.

50. Ibid.

51. CORCORAN Franck et al., "Inuit3D: An Interactive Virtual 3D Web Exhibition », Ottawa, NRC Publications Archive NPArC, 2002; GALANI Areti, «Mixed Reality Museum Visits: Using New Technologies to Support Co-visiting for Local and Remote Visitors », Museological Review, 2003, p. 1-15 ; RISVIC et al., « Interactive Digital Storytelling », art. cité. 
52. SCHWEIBENZ, «Le musée virtuel », art. cité.

53. RISVIC et al., « Interactive Digital Storytelling », art. cité.

54. Ibid.

55. NAEF, « Tourisme de mémoire », art. cité.

56. ETCHEVERRY Marc, "Le Musée national de Bosnie rouvre, un pied de nez aux divisions », RFI, 2015, en ligne: http://www.rfi.fr/zoom/20150914-bosnie-musee-national-divisions-ouverturemobilisation-citoyens (consulté en janvier 2019)

57. Ibid.

58. NAEF, « Tourisme de mémoire », art. cité, p. 78.

59. REKACEWICZ Philippe, "Bosnie, le partage de Dayton (21 septembre 1995)", Le Monde diplomatique, 1998, en ligne: https://www.monde-diplomatique.fr/cartes/bosniemdv1999 (consulté en novembre 2019).

60. NAEF, « Tourisme de mémoire », art. cité.

61. UNESCO, Culture: futur urbain, rapport mondial sur la culture pour le développement urbain mondial, Paris, rapport final, 2016.

62. KASSOUHA, « Tourisme(s) post-conflit », art. cité.

63. PETRITSCH Wolfgang, solioz Christophe, Ownership Process in Bosnia and Herzegovina, Baden Baden, Nomos Verlagsgesellschaft, 2003 ; NAEF, « Tourisme de mémoire », art. cité, p. 73.

64. GÜL Murat, DEE John, « Sarajevo - A City Profile », Cities, vol. 43, 2015, p. 152-166.

65. WASHBURN Doug, SINDHU Usman, «Helping CIOs Understand "Smart City" Initiatives", Forrester, 2010, p. 1-17.

66. Ibid.

67. UNDP, « "Sarajevo - Smart City Initiative" Launched ", art. cité.

68. HRNJIC Anes, šUTA Nedim, PILAY-VELIC Amila, «Innovation in Senior Tourism: Creating Energy Efficient and Health Supported Tourist Offer ", Review of Innovation and Competitiveness, vol. 2, $\mathrm{n}^{\circ} 4$, 2016, p. 5-18.

69. Ibid.

70. JANG Shawn, wU Chi-Mei Emily, "Travel Motivations and the Influential Factors: An Examination of Taiwanese Seniors ", Tourism Management, vol. 27, $n^{\circ} 2,2006$, p. 306-316 ; LE SERRE Delphine, LEGoHÉREL Patrick, WEBER Karin, «Motivations and Perceived Risks: A Cross-cultural Study », Journal of International Consumer Marketing, vol. 25, n² 2, 2013, p. 61-79; HRNJIC, šUTA, PILAYVELIC, « Innovation in Senior Tourism », art. cité.

71. IE, «Conférence - Ville intelligente: résilience et influence internationale», Centre de ressources et d'information sur l'intelligence économique et stratégique, 2019, en ligne : https:// portail-ie.fr/event/787/ville-intelligente-resilience-et-influence-internationale (consulté en juin 2019).

72. GUNDERSON, HOLLING (dir.), Panarchy: Understanding Transformations, op. cit.

73. Ibid.

74. TOUBIN et al., « La Résilience urbaine : un nouveau concept opérationnel », art. cité.

75. KURSAD ÖZLEN Muhammed, POTURAK Mersid, «Tourism in Bosnia and Herzegovina », Global Business and Economics Research Journal, vol. 2, $\mathrm{n}^{\circ}$ 6, 2013, p. 13-25.

76. TARDY Thierry, «L'ONU et la gestion des conflits yougoslaves (1991-1995): faillite d'une institution, faillite des États? », Relations internationales, vol. 4, $\mathrm{n}^{\circ}$ 4, 2006, p. 37-53.

77. Ibid.

78. TAINTER Joseph, The Collapse of Complex Societies, Cambridge, University Press, coll. «New Studies in Archaeology », 1990.

79. WALKER Jeremy, COOPER Melinda, «Genealogies of Resilience. From Systems Ecology to the Political Economy of Crisis Adaptations ", Security Dialogue, vol. 14, $\mathrm{n}^{\circ}$ 2, 2011, p. 1-28; QUENEAULT 
Béatrice, "La résurgence/convergence du triptyque "catastrophe-résilience-adaptation" pour (re)penser la "fabrique urbaine" face aux risques climatiques", Développement durable et territoires, vol. 5, $\mathrm{n}^{\circ}$ 3, 2014, p. 1-38.

80. WEF, «The Travel \& Tourism Competitiveness Report 2017. Paving the Way for a More Sustainable and Inclusive Future ", World Economic Forum, 2017, en ligne: http:// www3.weforum.org/docs/WEF_TTCR_2017_web_0401.pdf (consulté en janvier 2019).

81. Ibid.

82. WTTC, «Travel and Tourism Economic Impact 2017. Bosnia Herzegovina », World Travel Tourism Council, 2018, en ligne : https://www.wttc.org/-/media/files/reports/economic-impactresearch/countries-2017/bosniaherzegovina2017.pdf (consulté en janvier 2019).

\section{RÉSUMÉS}

Ces vingt dernières années, les technologies de l'information, la création de plateformes numérique de type peer-to-peer (Booking, Airbnb, Expedia) ou encore la réalité virtuelle au service des visites muséales et patrimoniales font exploser l'attractivité des destinations et les projets touristiques. Les projets touristiques basés sur le numérique et les nouvelles technologies sont en plein essor dans les Balkans, particulièrement en Bosnie-Herzégovine. En nous penchant sur différents projets technologiques et digitaux à visée touristique, nous verrons comment des acteurs locaux, privés, transnationaux et citoyens se sont emparés de ce projet pour tenter de le réaliser.

Over the last twenty years, information technologies, the creation of peer-to-peer digital platforms (Booking, Airbnb, Expedia) and virtual reality for museum and heritage visits have boosted the attractiveness of destination and tourism projects. The emergence of tourism based on digital and new technologies is booming in the Balkans, particularly in Bosnia-Herzegovina. We will look at different technological and digital projects for tourism purposes to see how local, private, transnational and citizen actors have tried to bring them into being.

\section{INDEX}

Mots-clés : tourisme, destination, résilience, technologies, numérique

Keywords : tourism, destination, resilience, technologies, digital

\section{AUTEUR}

\section{CYRIL MARTIN-COLONNA}

Candidat au doctorat en études urbaines, ESG-UQÀM

Martin.cyril[at]uqam.ca 